\title{
Temperature dependent fluorescence in disordered Frenkel chains: interplay of equilibration and local band-edge level structure
}

\author{
M. Bednarz, ${ }^{1}$ V.A. Malyshev, ${ }^{2}$ and J. Knoester ${ }^{1}$ \\ ${ }^{1}$ Institute for Theoretical Physics and Material Science Center, \\ University of Groningen, Nijenborgh 4, 9747 AG Groningen, The Netherlands \\ ${ }^{2}$ S.I. Vavilov State Optical Institute", Birzhevaya Liniya 12, 199034 Saint-Petersburg, Russia
}

(Dated: October 31, 2018)

\begin{abstract}
We model the optical dynamics in linear Frenkel exciton systems governed by scattering on static disorder and lattice vibrations, and calculate the temperature dependent fluorescence spectrum and lifetime. The fluorescence Stokes shift shows a nonmonotonic behavior with temperature, which derives from the interplay of the local band-edge level structure and thermal equilibration. The model yields excellent fits to experiments performed on linear dye aggregates.
\end{abstract}

PACS numbers: 71.35.Aa; 36.20.Kd; 78.30.Ly

Optical dynamics and emission of light in solid state systems are topics of considerable fundamental and technological importance. A variety of materials is currently studied as light emitting devices. Examples are semiconductor quantum wells and wires 1], conjugated polymers [2], and molecular aggregates [3]. Closely related is the quest for understanding the optical dynamics in photosynthetic antenna systems [4, 5]. The fundamental understanding and description of the emission properties of all these systems are challenging, because they involve a complicated interplay of processes. The emission is usually caused by excitons and is particularly sensitive to the scattering of these quasi-particles on static disorder, which may lead to their localization, and lattice vibrations. The latter results in vibronic relaxation and hopping of the excitation between (localized) exciton states, which gives rise to spatial and spectral diffusion. These diffusion processes compete with spontaneous emission and nonradiative loss. Depending on the details of this competition, the excitons may or may not reach thermal equilibrium before emission occurs. Temperature plays an important role in this competition. The thermal destruction of cooperative emission in molecular aggregates [6], quantum wells [7], and quantum wires [8, 9] is a wellknown example of an interesting temperature dependent effect. The Stokes shift of the exciton fluorescence may exhibit interesting thermal behavior as well 10, 11].

In this Letter, we theoretically study the exciton dynamics and the resulting emission properties of linear chains carrying small-radius excitons. We account for both exciton localization by static disorder and scattering of the excitons on lattice vibrations. The interplay of nonequilibrium conditions and the local band-edge level structure of disordered chains leads to interesting effects in the emission. A comparison to experiment is made.

We consider a chain of $N$ molecules described by the Frenkel exciton Hamiltonian

$$
H=\sum_{n=1}^{N} \epsilon_{n}|n\rangle\left\langle n\left|+\sum_{n, m}^{N} J_{n m}\right| n\right\rangle\langle m|,
$$

where $\epsilon_{n}$ is the excitation energy of the $n$th molecule and $|n\rangle$ denotes the state in which molecule $n$ is excited and all others are in their respective ground states. In order to model the effect of random solvent shifts, the $\epsilon_{n}$ are assumed to be mutually uncorrelated Gaussian stochastic variables with mean $\epsilon_{0}$ and standard deviation $\sigma$. The hopping integrals $J_{n m}$ are nonrandom and originate from dipolar coupling: $J_{n m}=-J /|n-m|^{3}, J_{n n} \equiv 0$. Here, $J$ is positive, implying that the optically allowed exciton states reside at the bottom of the exciton band.

We will assume that the coupling of the excitons to lattice vibrations is weak. It is then appropriate to use a basis of eigenstates of $H$. This basis consists of $N$ exciton states, which are labeled by $\nu=1, \ldots, N$. The energy of the $\nu$ th exciton, $E_{\nu}$, is the $\nu$ th eigenvalue of the $N \times N$ matrix $H_{n m}=\langle n|H| m\rangle$, while the $n$th component of the corresponding eigenvector, $\varphi_{\nu n}$, gives the amplitude of the $\nu$ th exciton state on molecule $n:|\nu\rangle=\sum_{n=1}^{N} \varphi_{\nu n}|n\rangle$. The disorder $\sigma$ leads to localization of the eigenstates and gives rise to a tail in the density of states (DOS) below the bare band edge 12, 13. This tail is of particular interest for optical properties, as the states residing in it carry most of the oscillator strength, $F_{\nu}=\left|\sum_{n} \varphi_{\nu n}\right|^{2}$. While the DOS accumulated over the entire chain is a smooth function of energy, locally it exhibits structure 14]. This local level structure is of key importance to the low-temperature dynamics and is described below.

The exciton states in the tail of the DOS are localized on finite segments, which near the band edge have a typical size $N^{*}$ (see Fig. 11). Within its own segment, each one of these states is the lowest-energy eigenstate. They have wave functions without nodes in the segments and carry an oscillator strength $F_{\nu} \approx N^{*}$. Their spontaneous emission rates, $\gamma_{\nu}=\gamma_{0} F_{\nu}$ (with $\gamma_{0}$ the emission rate of a single molecule), thus scale like $N^{*}$. This is referred to as superradiant enhancement [6]. Bottom states on neighboring segments have small overlap. Often a second, higher-energy, exciton state may be distinguished on a segment, separated from the bottom state by an energy of the order of $3 \pi^{2} J / N^{* 2}$, as is dictated by energy 


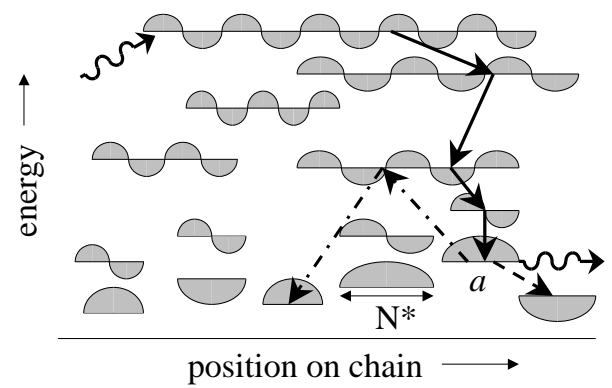

FIG. 1: Schematic picture of the localized exciton wave functions near the lower band edge in a disordered linear chain. Also indicated are the optical pumping and emission processes (wavy arrows) and various intraband relaxation processes (dashed and dash-dotted arrows).

quantization. These states have one node within the segments and small oscillator strengths. Still higher local states are usually delocalized over two or more segments and carry negligible oscillator strengths. The local level structure is washed out in the average over the entire chain due to the fact that the distribution of energies of the bottom segment states also has a width of the order of $3 \pi^{2} J / N^{* 2}$ [14].

Weak exciton-phonon coupling leads to scattering between the exciton eigenstates. To describe this dynamics, we will use the Pauli master equation for the populations, $P_{\nu}(t)$, of the localized exciton states:

$$
\dot{P}_{\nu}=R_{\nu}(t)-\gamma_{\nu} P_{\nu}+\sum_{\mu=1}^{N}\left(W_{\nu \mu} P_{\mu}-W_{\mu \nu} P_{\nu}\right) .
$$

Here, the dot denotes the time derivative, $R_{\nu}(t)$ is the optical pumping rate, $\gamma_{\nu}$ is the above defined spontaneous emission rate, and $W_{\nu \mu}$ is the transition rate from the localized state $\mu$ to the state $\nu$ induced by the excitonphonon scattering. For the latter, we will use

$$
W_{\nu \mu}=W_{0} S\left(E_{\nu}-E_{\mu}\right) G\left(E_{\nu}-E_{\mu}\right) \sum_{n=1}^{N} \varphi_{\nu n}^{2} \varphi_{\mu n}^{2}
$$

where the constant $W_{0}$ characterizes the scattering, the sum over sites is the overlap integral of exciton probabilities for the states $\mu$ and $\nu$, and $S\left(E_{\nu}-E_{\mu}\right)$ describes that part of the $\left|E_{\nu}-E_{\mu}\right|$ dependence of $W_{\nu \mu}$ that derives from the energy dependence of the exciton-phonon coupling constant and the phonon density of states. Finally, $G(E)=n(E)$ if $E>0$ and $G(E)=1+n(-E)$ if $E<0$, with $n(\Omega)=\left[\exp \left(\Omega / k_{\mathrm{B}} T\right)-1\right]^{-1}$ the mean occupation number of a phonon state with the energy $\Omega$. Equation (3), which meets the principle of detailed balance, is the generic result from first-order perturbation theory in the exciton-phonon interaction [15, 16, 17].

We have numerically simulated the above model of intra-band exciton dynamics for scattering on acoustic phonons of the chains' host medium. We have set
$S=\left|E_{\nu}-E_{\mu}\right| / J$, which properly accounts for a decrease of the coupling of excitons to acoustic phonons at small wave vectors and prevents the divergence of $W_{\nu \mu}$ at small energy differences. We will illustrate the salient results of our model by using parameters that are relevant to molecular aggregates of the dye THIATS: $\epsilon_{0}=18138$ $\mathrm{cm}^{-1}, J=740 \mathrm{~cm}^{-1}$, and $\gamma_{0}=(2 / 31) \times 10^{8} \mathrm{~s}^{-1}[11,18$. These parameters are fixed; the two free parameters are $\sigma$ and $W_{0}$, for which we will use the values that yield the best fit to experimental data: $\sigma=0.23 \mathrm{~J}$ and $W_{0}=30 \mathrm{~J}$, respectively (vide infra). A more elaborate analysis of our model, covering a wider range of values for $\sigma$ and $W_{0}$, will be discussed elsewhere [19]. The motivation to focus on the example of THIATS aggregates, is that a large number of temperature dependent spectroscopic data are known for these systems 11, 20]. We will limit our attention to temperatures small compared to the Davydov splitting of $3000 \mathrm{~cm}^{-1}$ that exists for these aggregates between the so-called $\mathrm{J}$ band at the lower exciton band edge and the so-called $\mathrm{H}$ band at the upper band edge [11, 18]. For these temperatures our effective model with one molecule per unit cell may be used [21]. We have used a chain size of $N=500$ in our simulations, which far exceeds the typical band-edge exciton size $N^{*}=30$ for $\sigma=0.23 J$.

We will first address the absorption and steady-state emission spectra, which are calculated through

$$
\begin{gathered}
A(E)=\frac{1}{N}\left\langle\sum_{\nu=1}^{N} \gamma_{\nu} \delta\left(E-E_{\nu}\right)\right\rangle, \\
I^{\mathrm{st}}(E)=\frac{1}{N}\left\langle\sum_{\nu} \gamma_{\nu} P_{\nu}^{\mathrm{st}} \delta\left(E-E_{\nu}\right)\right\rangle,
\end{gathered}
$$

respectively. Here, the angular brackets denote the average over the disorder realizations. Furthermore, $P_{\nu}^{\text {st }}$ is the steady-state solution of the master equation Eq. (2) with the source term given by $R_{\nu}(t)=F_{\nu}$ if $E_{\nu}$ falls inside a narrow window in the blue wing of the $\mathrm{J}$ band and $R_{\nu}=0$ otherwise. This blue-tail excitation condition agrees with common experimental conditions.

The spectra calculated for $T=10 \mathrm{~K}$ are shown by solid lines in Fig. 2 together with the J band and fluorescence spectrum measured for THIATS aggregates at the same temperature (dots) 11. Clearly, both experimental spectra are fitted very well within our model. The main deviations occur in the tails, which are quite sensitive to the precise nature of the disorder (diagonal versus off-diagonal disorder, no perfect alignment of molecular dipoles). We note that the fluorescence band is shifted to the red (Stokes shift) and narrowed with respect to the $\mathrm{J}$ band. Keeping in mind that the absorption band is dominated by the set of superradiant bottom states of the various localization segments on the chain, this is an indication that after the fast initial relaxation to a particular 


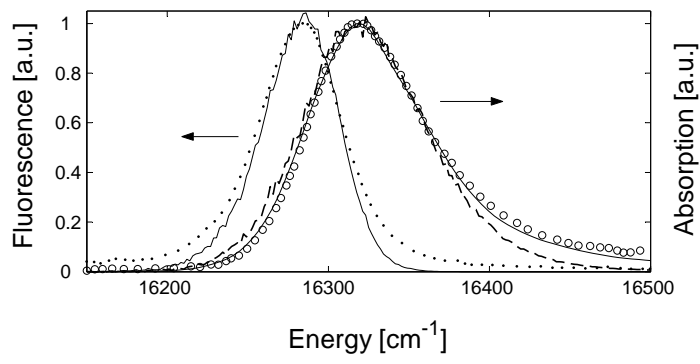

FIG. 2: Calculated absorption and fluorescence spectra (solid lines) at $10 \mathrm{~K}$ for the model parameters described in the text, compared to the $\mathrm{J}$ band (o) and fluorescence spectrum (•) for THIATS aggregates measured at $T=10 \mathrm{~K}$ [11]. The dashed line is the fluorescence spectrum calculated for the same parameter set, except that now $W_{0}=10^{-3} \mathrm{~J}$.

bottom state (e.g., the level $a$ in Fig. 1), the excitons have a chance to relax to even lower states. This is a signature of migration to other localization segments, a process which at low temperatures is hindered by the small overlap between exciton wave functions of different segments. The fact that, in spite of this, migration may occur before radiative emission takes place, is due to the large value of $W_{0}$, which brings the system into a regime that we refer to as the fast-intersegment-relaxation limit. To illustrate the role of $W_{0}$, we also present in Fig. 2 (dashed line) the simulated fluorescence spectrum for $W_{0}=10^{-3} \mathrm{~J}$ (all other parameters as above). In this slow-relaxation limit the fluorescence spectrum closely follows the absorption band and no Stokes shift occurs.

At zero temperature, the possibility for the excitation to migrate to other localization segments, after the initial relaxation process, is very small. The reason is that the migration may then only occur via a jump to a lower exciton state on a neighboring segment, for which at least some (albeit small) wave function overlap exists (cf. the process denoted by the dashed arrow in Fig. 11). However, the probability of finding a lower exciton state on a neighboring segment is small, because the initial state already lies in the tail of the DOS. Therefore, in practice, low-temperature exciton migration is limited to at most one jump. As a consequence of this blockade for diffusion, there is not much opportunity for the excitation to explore the full DOS during its lifetime and to equilibrate to the lowest state on the disordered chain. Thus, even for large $W_{0}$, the zero temperature Stokes shift is limited to values of the order of the $\mathrm{J}$ band width.

Upon increasing the temperature, higher states may thermally be excited and one expects the Stokes shift to decrease and eventually vanish. This is the generic behavior for single molecules. Our simulations show that at elevated temperatures this also occurs for the exciton system (Fig. 3(a)). However, our results also reveal a counter-intuitive intermediate regime, where the Stokes shift increases with temperature. This nonmono-
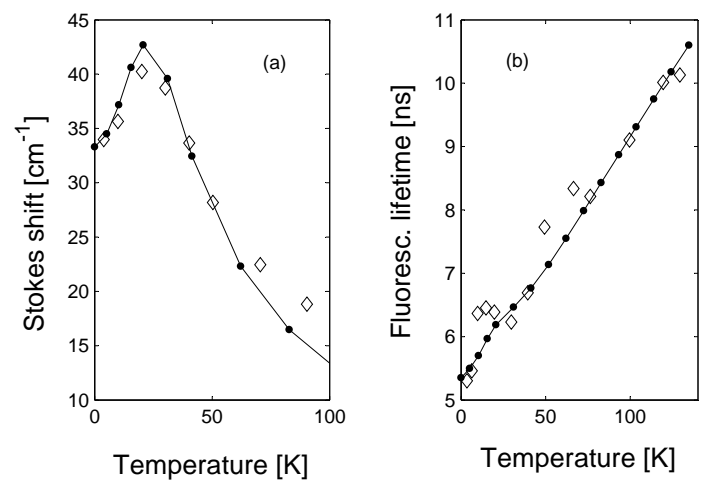

FIG. 3: (a) Calculated Stokes shift of the fluorescence spectrum as a function of temperature for the parameter set described in the text $(\bullet)$. The solid line is a guide to the eye. Diamonds mark the measured Stokes shift for THIATS aggregates reported in Ref. [11]. (b) As in (a), for the fluoresecence lifetime instead of the Stokes shift. Diamonds are the experimental data from Ref. [20].

tonic behavior is intimately related to the interplay of equilibration and the local level structure near the exciton band edge. Upon slightly increasing the temperature from zero, a small fraction of the population is thermally raised from the bottom state of a segment to a higher exciton state that overlaps with the same segment. As this higher state generally overlaps with more segments, this provides a means of distributing the population over two or more neighboring segments. The total process is denoted by the dash-dotted arrows in Fig. 1 This overlap-assisted activated migration is slow, but it allows the excitation to probe more segments for a low-energy position and circumvents to some extent the blockade for migration which occurs at $T=0 \mathrm{~K}$. Hence, the excitons get closer to thermal equilibrium, which at low temperatures leads to a larger Stokes shift. Such anomalous low-temperature behavior has also been predicted and observed for Wannier excitons migrating between local potential minima in narrow quantum wells [10].

As we also see in Fig. 3(a), our numerical results, obtained for the same parameter set as was used to fit the low-temperature spectra, agree very well with the experimental data for THIATS aggregates reported in Ref. [1]. This gives strong evidence for the validity of our dynamic model and the concept of overlap-assisted thermallyactivated intersegment hopping. Using the typical local level separation $3 \pi^{2} J / N^{* 2}$ and the superradiant emission rate $N^{*} \gamma_{0}$, it may be shown that the temperature interval over which the Stokes shift behaves in an anomalous way (i.e., increases with $T$ ) scales roughly linearly with the absorption line width and only weakly (inverse logarithmically) depends on the ratio $W_{0} / \gamma_{0}$ [19].

We finally turn to the lifetime of the total fluorescence intensity after pulsed excitation. For the generally nonexponential intensity trace $I(t)=-\left\langle\sum_{\nu} \dot{P}_{\nu}(t)\right\rangle$, we de- 
fine the lifetime as its first moment, i.e., the expectation value of the photon emission time: $\tau=\int_{0}^{\infty}\left\langle\sum_{\nu} P_{\nu}(t)\right\rangle \mathrm{d} t$ [17]. Here, $P_{\nu}(t)$ is the solution of Eq. (2) with $R_{\nu}(t)=$ $c F_{\nu} \delta(t)$ if $E_{\nu}$ falls in a narrow window in the blue tail of the absorption band and $R_{\nu}(t)=0$ otherwise. The normalization constant $c$ is such that directly following the excitation pulse the total population on the chain, $\sum_{\nu} P_{\nu}$, equals unity.

The generic behavior of the lifetime as a function of temperature is well known. At low $T$, the decay is dominated by the superradiant bottom states of the localization segments, leading to a fluorescence lifetime of the order of $1 / N^{*} \gamma_{0}$. With increasing $T$, the exciton population is shared by the superradiant states as well as an increasing number of higher-lying dark states. This leads to an increase of the fluorescence lifetime, as has been observed for various exciton systems [6, 7, 8, 9, 20, 22]. For THIATS aggregates the lifetime shows a nearly linear temperature dependence from 0 to $130 \mathrm{~K}$ 20] (see Fig. 3] b), diamonds). For BIC aggregates a similar linear increase was observed and, based on the flat nature of the DOS for ideal two-dimensional systems, this was put forward as evidence for a two-dimensional structure of these aggregates [22]. It turns out, however, that the interplay of the (local) DOS and equilibration in our one-dimensional model with disorder may equally well explain the quasilinear behavior. The solid line in Fig. 3(b) gives our simulated results, obtained for the same parameters that were used to fit the low-temperature spectra of THIATS. We conclude that the model, without any new parameters, yields an excellent fit to the experimental data. We also mention that at small $W_{0}$ (slow relaxation), the linear temperature dependence may be preceded by a lowtemperature plateau [19], which is the type of behavior that is observed for aggregates of pseudo-isocyanine [6].

In summary, we have analyzed the temperature dependence of the fluorescence in one-dimensional disordered exciton systems and obtained excellent fits to experimental data for aggregates of the THIATS dye molecules. As far as we are aware, this is the first model calculation that yields such good agreement with a variety of fluorescence experiments on linear dye aggregates. Previous calculations focused only on the temperature dependent lifetime and did not account for exciton localization by disorder [23]. Our approach, based on the master equation with perturbatively calculated rates $W_{\nu \mu}$, is consistent as long as the rates for scattering between the optically dominant states do not exceed their energy separation. For the parameters used for THIATS we find that, in spite of the seemingly large value of $W_{0}$, at low temperature this criterion is just obeyed, due to the small overlap integral $\sum_{n=1}^{N} \varphi_{\nu n}^{2} \varphi_{\mu n}^{2} \sim 1 / N^{*}$ for the exciton wave functions near the band edge. With increasing temperature, the scattering rates increase and around $100 \mathrm{~K}$ they lead to a visible contribution to the absorption line width (homogeneous broadening) [1]. At such high temperatures the master equation description breaks down and the full exciton density matrix must be considered instead [4].

V.A.M. acknowledges support from la Universidad Complutense at the initial stage of this work.

[1] M.S. Gudiksen, L.J. Lauhon, J. Wang, D.C. Smith, and C.M. Lieber, Nature 415, 617 (2002).

[2] R. Gupta, J.Y. Park, V.I. Srdanov, and A.J. Heeger, Synth. Met. 132, 105 (2002).

[3] F.C. Spano, Chem. Phys. Lett. 331, 7 (2000).

[4] T. Renger and V. May, Phys. Rev. Lett. 84, 5228 (2000).

[5] G. Trinkunas, J.L. Herek, T. Polivka, V. Sundström, and T. Pullerits, Phys. Rev. Lett. 86, 4167 (2001); C. Hoffman, M. Ketelaars, M. Matsushita, H. Michel, T.J. Aartsma, and J. Köhler, Phys. Rev. Lett. 90, 013004 (2003).

[6] S. de Boer and D. A. Wiersma, Chem. Phys. Lett. 165, 45 (1990)

[7] J. Feldmann, G. Peter, E.O. Göbel, P. Dawson, K. Moore, C. Foxon, R.J. Elliott, Phys. Rev. Lett. 59, 2337 (1987).

[8] D.S. Citrin, Phys. Rev. Lett. 69, 3393 (1992).

[9] D.Y. Oberli, M.A. Dupertuis, F. Reinhardt, and E. Kapon, Phys. Rev. B 59, 2910 (1999).

[10] R. Zimmerman and E. Runge, phys. stat. sol. (a) 164, 511 (1997); M. Grassi Alessi et al., Phys. Rev. B 61, 10985 (2000).

[11] I.G. Scheblykin, O.Yu. Sliusarenko, L.S. Lepnev, A.G. Vitukhnovsky, and M. Van der Auweraer, J. Phys. Chem. B 105, 4636 (2001).

[12] M. Schreiber and Y. Toyozawa, J. Phys. Soc. Jpn. 51, 1528 (1982); ibid. 1537 (1982).

[13] H. Fidder, J. Knoester, and D. A. Wiersma, J. Chem. Phys. 95, 7880 (1991).

[14] V. Malyshev and P. Moreno, Phys. Rev. B 51, 14587 (1995); A. V. Malyshev and V. A. Malyshev, Phys. Rev. B 63, 195111 (2001).

[15] J.A. Leegwater, J.R. Durrant, and D.R. Klug, J. Phys. Chem. 101, 7205 (1997).

[16] M. Shimizu, S. Suto, and T. Goto, J. Chem. Phys. 114, 2775 (2001).

[17] M. Bednarz, V.A. Malyshev, and J. Knoester, J. Chem. Phys. 117, 6200 (2002).

[18] D.M. Basko, A.N. Lobanov, A.V. Pimenov, A.G. Vitukhnovsky, Chem. Phys. Lett. 369, 192 (2003).

[19] M. Bednarz, V.A. Malyshev, and J. Knoester, J. Chem. Phys. (submitted).

[20] I. G. Scheblykin, M. M. Bataiev, M. Van der Auweraer, A. G. Vitukhnovsky, Chem. Phys. Lett. 316, 37 (2000).

[21] This effective model requires multiplying the real molecular oscillator strength by the fraction of the total oscillator strength that is accumulated in the $\mathrm{J}$ band (i.e., = $1 / 31[11]$ ), as was done in our choice of $\gamma_{0}$.

[22] V. F. Kamalov, I. A. Struganova, and K. Yoshihara, J. Phys. Chem. 100, 8640 (1996).

[23] F. C. Spano, J. R. Kuklinsky, and S. Mukamel, Phys. Rev. Lett. 65, 211 (1990); E. O. Potma and D. A. Wiersma, J. Chem. Phys. 108, 4894 (1998). 